\title{
NRXN1 is associated with enlargement of the temporal horns of the lateral ventricles in psychosis
}

\author{
Ney Alliey-Rodriguez $\mathbb{0}^{1}$, Tamar A. Grey², Rebecca Shafee ${ }^{3,4}$, Huma Asif', Olivia Lutz $\mathbb{0}^{5}$, Nicolas R. Bolo5, \\ Jaya Padmanabhan $\mathbb{0}^{5}$, Neeraj Tandon ${ }^{5}$, Madeline Klinger ${ }^{1}$, Katherine Reis ${ }^{1}$, Jonathan Spring ${ }^{6}$, Lucas Coppes ${ }^{1}$, \\ Victor Zeng ${ }^{7}$, Rachal R. Hegde $\mathbb{0}^{8}$, Dung T. Hoang ${ }^{7}$, Deepthi Bannai ${ }^{8}$, Uzma Nawaz ${ }^{8}$, Philip Henson7, Siyuan Liu', \\ Diane Gage ${ }^{10}$, Steven McCarroll1 ${ }^{10}$, Jeffrey R. Bishop ${ }^{11}$, Scot Hill ${ }^{12}$, James L. Reilly ${ }^{13}$, Rebekka Lencer $\mathbb{E}^{14}$, \\ Brett A. Clementz ${ }^{15}$, Peter Buckley ${ }^{16}$, David C. Glahnn" ${ }^{17}$, Shashwath A. Meda ${ }^{17}$, Balaji Narayanan ${ }^{17}$, Godfrey Pearlson ${ }^{17}$, \\ Matcheri S. Keshavan, Elena I. Ivleva ${ }^{18}$, Carol Tamminga ${ }^{18}$, John A. Sweeney ${ }^{18}$, David Curtis $\mathbb{D}^{19}$, Judith A. Badner ${ }^{20}$, \\ Sarah Keedy ${ }^{1}$, Judith Rapoport ${ }^{9}$, Chunyu Liu (D) ${ }^{21}$ and Elliot S. Gershon (iD) ${ }^{1,22}$
}

\begin{abstract}
Schizophrenia, Schizoaffective, and Bipolar disorders share behavioral and phenomenological traits, intermediate phenotypes, and some associated genetic loci with pleiotropic effects. Volumetric abnormalities in brain structures are among the intermediate phenotypes consistently reported associated with these disorders. In order to examine the genetic underpinnings of these structural brain modifications, we performed genome-wide association analyses (GWAS) on 60 quantitative structural brain MRI phenotypes in a sample of 777 subjects (483 cases and 294 controls pooled together). Genotyping was performed with the Illumina PsychChip microarray, followed by imputation to the 1000 genomes multiethnic reference panel. Enlargement of the Temporal Horns of Lateral Ventricles (THLV) is associated with an intronic SNP of the gene NRXN1 (rs12467877, $P=6.76 \mathrm{E}-10$ ), which accounts for $4.5 \%$ of the variance in size. Enlarged THLV is associated with psychosis in this sample, and with reduction of the hippocampus and enlargement of the choroid plexus and caudate. Eight other suggestively significant associations $(P<5.5 \mathrm{E}-8)$ were identified with THLV and 5 other brain structures. Although rare deletions of NRXN1 have been previously associated with psychosis, this is the first report of a common SNP variant of NRXN1 associated with enlargement of the THLV in psychosis.
\end{abstract}

\section{Introduction}

There is ample evidence of partially overlapping brain morphology abnormalities in schizophrenia (SZ), schizoaffective disorder (SAD), and bipolar disorder $(\mathrm{BD})^{1-3}$. These disorders also have a shared set of genetic loci associated with them which can generate diverse disease phenotypes (pleiotropism), and a partially shared

Correspondence: Ney Alliey-Rodriguez (nalliey@bsd.uchicago.edu) or

Elliot S. Gershon (egershon@yoda.bsd.uchicago.edu)

'University of Chicago, Department of Psychiatry and Behavioral

Neurosciences, Chicago, USA

${ }^{2}$ Massachusetts Institute of Technology, Cambridge, USA

Full list of author information is available at the end of the article. polygenic diathesis ${ }^{4,5}$, as well as shared behavioral features, including psychosis in some BD patients. Although large samples of patients with psychotic mental disorders and controls have undergone GWAS, the phenotypes in these studies have been mostly restricted to diagnostic categories $^{4,6}$, and most studies of intermediate phenotypes associated with psychosis reported are based on smaller samples $^{7-10}$. Gottesman proposed the term endophenotype for sub-phenotypes observable in patients that might prove more tractable to genetic analysis ${ }^{11,12}$, and independently Gershon et al. ${ }^{13,14}$ proposed a similar idea. Following these concepts, the Bipolar and Schizophrenia Network for Intermediate Phenotypes study (B-SNIP)

\section{(c) The Author(s) 2019}

(c) (i) Open Access This article is licensed under a Creative Commons Attribution 4.0 International License, which permits use, sharing, adaptation, distribution and reproduction c. in any medium or format, as long as you give appropriate credit to the original author(s) and the source, provide a link to the Creative Commons license, and indicate if changes were made. The images or other third party material in this article are included in the article's Creative Commons license, unless indicated otherwise in a credit line to the material. If material is not included in the article's Creative Commons license and your intended use is not permitted by statutory regulation or exceeds the permitted use, you will need to obtain permission directly from the copyright holder. To view a copy of this license, visit http://creativecommons.org/licenses/by/4.0/. 
collected multiple phenotypes on each studied individual, aimed to identify biomarkers of psychosis and their genetic correlations, which can help elucidate mechanisms of the disorders, and lead to improved classifications and personalized treatment ${ }^{15}$. Previous neuroimaging studies found enlargement of the ventricular system in bipolar disorder and schizophrenia ${ }^{16,17}$, and the temporal (inferior) horns of the lateral ventricles (THLV) were also reported enlarged in patients with these disorders ${ }^{18}$. Here we present genome-wide association analyses of brain imaging in 777 B-SNIP patients with psychosis and healthy controls. Although this sample size is smaller than current large case-control studies, the power to detect associations using quantitative phenotypes is higher than with dichotomous traits, and pooling ill subjects with normal controls to analyze quantitative traits has the advantage of a wider range in the observed phenotypes, which translates into higher power to detect genetic associations ${ }^{19}$. As noted by Dahl et al. ${ }^{20}$, deep phenotyping with simultaneous genome-wide analyses can serve as a discovery tool for previously unsuspected relationships of phenotypic traits with each other, and with shared molecular events. In this study we found a significant association between the THLV volume and a common intronic SNP of the gene Neurexin 1 (NRXN1). NRXN1 is known to be involved in brain development and function. These facts support the potential role of this common variant of NRXN1 in psychosis.

\section{Methods}

The Bipolar and Schizophrenia Network for Intermediate Phenotypes (B-SNIP) studies individuals with schizophrenia (SZ), schizoaffective disorder (SAD), bipolar disorder with psychosis (BD), and healthy controls. Data were collected between 2008 and 2012 at six study sites in the USA, with IRB approval at each participating institution. Details of phenotype collection are described in Tamminga el al. ${ }^{21,22}$, including standardization of phenotyping methods across all the collaborating sites. Here we present genome-wide association analyses of structural brain MRI data from 59 brain volumes plus the whole brain gray matter density, studied in 483 cases (169 BD, 127 SAD, and 187 SZ) and 294 healthy controls. Sample demographics are shown in Table 1. A total of 60 quantitative traits (listed in Supplementary Material) from these 777 cases and controls pooled together are analyzed here as phenotypes in genome-wide association studies (GWAS). Diagnostic categories were not used as phenotypes or as covariates in GWAS, except for post-hoc analyses to check on phenotypic associations with case-control status in this dataset, and whether our top SNP was associated with disease. The rationale to pool cases and controls is that in the study of quantitative traits when the phenotype is associated with disease there will be greater phenotypic variation and thus more power to detect association. These associations could help to explain part of the shared pathophysiology of these disorders. Including each diagnostic category as a covariate would ignore the genetic intercorrelation of the diagnoses, and detract from estimates of genetic associations with the tested phenotypes.

\section{Phenotypes: Image processing}

Structural T1-weighted 3D magnetization-prepared rapid gradient echo (MPRAGE) scans were acquired at six sites: Boston (3.0 T, GE Signa), Detroit (3.0 T, Siemens Allegra), Baltimore (3.0 T, Siemens Trio trim), Hartford (3.0 T, Siemens Allegra), Dallas (3.0 T, Philips) and Chicago (3.0 T, GE Signa). The scans ( $\mathrm{TR}=6.7 \mathrm{msec}, \mathrm{TE}=3.1 \mathrm{msec}, 8^{\circ}$ flip angle, $256 \times 240$ matrix size, total scan duration $=$ 10:52.6 min, 170 sagittal slices, $1 \mathrm{~mm}$ slice thickness, $1 \times 1 \times$ $1.2 \mathrm{~mm}^{3}$ voxel resolution) were acquired following the Alzheimer's Disease Neuroimaging Initiative (ADNI) protocol (http://adni.loni.usc.edu/methods/documents/mriprotocols/). Scans were visually assessed for artifacts and processed using Freesurfer 6.0 (https://surfer.nmr.mgh. harvard.edu//23 using Scientific Linux 7.5. The scans went through first-level auto-reconstruction to undergo

Table 1 Sample Demographics

\begin{tabular}{|c|c|c|c|c|c|c|}
\hline & \multirow[b]{2}{*}{$N$} & \multirow{2}{*}{$\begin{array}{l}\text { Age } \\
\text { Mean } \pm \text { SD }\end{array}$} & \multirow{2}{*}{$\begin{array}{l}\text { Sex } \\
\text { Female \% }\end{array}$} & \multicolumn{3}{|c|}{ Self-reported Ethnicity } \\
\hline & & & & Caucasian \% & African-American $\%$ & Others \% \\
\hline Controls & 294 & $38.19 \pm 12.48$ & 51.36 & 67.69 & 26.87 & 5.44 \\
\hline \multicolumn{7}{|l|}{ Cases } \\
\hline SZ & 187 & $34.99 \pm 12.43$ & 35.29 & 46.52 & 45.45 & 8.02 \\
\hline SAD & 127 & $35.94 \pm 11.87$ & 55.12 & 52.76 & 41.73 & 5.51 \\
\hline $\mathrm{BD}$ & 169 & $36.46 \pm 13.13$ & 69.23 & 76.92 & 19.53 & 3.55 \\
\hline All Cases & 483 & $35.75 \pm 12.53$ & 52.38 & 58.80 & 35.40 & 5.80 \\
\hline Total Sample & 777 & $36.67 \pm 12.56$ & 51.99 & 62.16 & 32.18 & 5.66 \\
\hline
\end{tabular}


registration in standard space, motion correction and skull striping. Trained raters edited the images to remove dura, sinuses and blood vessels that could interfere with segmentation. Scans were run through second and third level auto-reconstruction to segment gray-white matter, reviewed by two independent raters and removed if motion interfered with segmentation. A total of 777 scans were retained for analysis and registered to the Desikan/Killiany $\operatorname{atlas}^{24}$. For this study we used only FreeSurfer volumetric variables, joining left and right hemisphere volumes into single bilateral variables. The whole brain gray matter density was calculated using Voxelbased Morphometry Toolbox for SPM8 as in Ivleva et al. ${ }^{25}$.

\section{Genotypes}

Genotypes were assessed from blood DNA at the Broad Institute using the Illumina Infinium Psycharray (PsychChip) (Illumina Inc., San Diego, CA, USA), which contains a total of 588,454 SNP markers, including 50,000 specific genetic markers for neuropsychiatric disorders. PsychChip genotype calls were processed through a custom pipeline at the Broad Institute designed to merge calls from 3 different algorithms (GenCall, Birdseed and $\mathrm{zCall}$ ), in order to maximize reliability and usability of rare markers (https://sites.google.com/a/ broadinstitute.org/psych-chip-resources/genotypecalling). We used programs PREST-plus ${ }^{26}$ and $\mathrm{KING}^{27}$ in order to remove individuals with 3rd degree or closer kinship. Pre-Imputation QC applied to PsychChip genotypes included filtering by Call Rate $>98 \%$ by SNP and $>$ $98 \%$ by sample, HWE $P$-value $>1 \mathrm{E}-06$ in controls, Inbreeding Coefficient $\left(-0.2>F_{-}\right.$Het $\left.>0.2\right)$, exclusion of monomorphic markers, Sex Check using X-chromosome heterozygosity and Y-chromosome call rate (all samples with sex check mismatch were dropped), and Minor Allele Frequency $(\mathrm{MAF})>0.01$.

\section{Genotype Imputation}

PsychChip genotypes were imputed to the 1000 Genomes phase 1 multiethnic reference panel ${ }^{28}$ using IMPUTE2 and HAPI-UR for pre-phasing ${ }^{29-31}$. Chromosomes were phased separately, and then divided into 5 megabase pair chunks for imputation. Poorly imputed SNPs (those with information score $<0.5$ ) were filtered out post-imputation. Imputed genotype probabilities with an uncertainty $<0.1$ were transformed to hard calls using PLINK 1.9. The final imputed genotype set contained 30 million autosomal markers, reduced to 4,322,238 variants after filtering for missingness by marker $<0.05$, missingness by individual sample $<0.02$, and MAF $>0.05$, in order to work with common variants only, given the limited power to detect rare events with our sample size (see power analysis in Suppl. Material).

\section{GWAS analyses}

GWAS analyses were performed using PLINK 1.9 linear regression model ${ }^{32,33}$ on the Bionimbus protected cloud of the Open Science Data Cloud servers at the University of Chicago (http://www.opensciencedatacloud.org) ${ }^{34}$. Genetic locations refer to the human genome GRCh37/ hg19 build, gene mapping was done using the UCSC genome browser ${ }^{35}$, and graphics generated with Manhattan Plotter, FUMA ${ }^{36}$ and LocusZoom ${ }^{37}$. Q-Q plots and calculated lambdas were used to examine possible inflation in GWAS results. We corrected for population stratification and admixture using the method of Price et al. $^{38}$; we used the first two eigenvectors from principal component analysis on the genotypes as covariates, which captured the majority of the ethnic-related variance (see Supplementary Material). Sex and age were used as covariates in all our analyses, and the total intracranial volume was also used as a covariate except for the association with itself as phenotype. Covariates used for gray matter density GWAS were age, sex, handedness and two PCA eigenvectors from genotypes.

Post-GWAS eQTL and functional genomic analyses were performed on FUMA version 1.3.4 (K. Watanabe, http://fuma.ctglab.nl//36, using data from The GenotypeTissue Expression (GTEx) project version 7 (https:// gtexportal.org) $)^{39}$ and the BrainSpan Atlas of the Developing Human Brain (http://www.brainspan.org//) ${ }^{40}$. The UK Brain Expression Consortium database (http:// http:// www.braineac.org $/)^{41}$ was also consulted for eQTLs of our top SNP markers.

\section{Significance Thresholds}

We calculated the GWAS statistical significance threshold for a single phenotype as $P<5.5 \mathrm{E}-08$ using the $\mathrm{Li}$ et al. method based on independent LD blocks from our genotypes ${ }^{42}$. In the context of multiple phenotype analyses reported, this is our threshold for suggestive significance. Many of the studied phenotypes are correlated, and as Bonferroni correction assumes independence of the tests, using it would increase the type-II error. We followed the $\mathrm{Li}$ and Ji method based on spectral decomposition of the phenotype correlation matrix to calculate the effective number of independent phenotype tests ${ }^{43}$. Thus, our significance threshold after multiple test correction was $5.5 \mathrm{E}-08 / 27$ independent effective tests $=P<2.04 \mathrm{E}-09$.

\section{Additional phenotypic associations}

Phenotypic associations were tested using analysis of variance (ANOVA) and partial correlations, calculated with IBM SPSS Statistics version 24.

\section{Results}

We identified a significant genome-wide association after multiple test correction between the combined 
Table 2 Genome-Wide Significant and Suggestive Association results

\begin{tabular}{|c|c|c|c|c|c|c|c|c|}
\hline Phenotype & Chr & Band & Base Pair & SNP marker & Allele & Beta & $P$ & Gene \\
\hline Temporal Horn of Lateral Ventricle & 2 & p16.3 & $50,368,229$ & rs12467877 & $\mathbf{T}$ & 184.9 & $6.76 \mathrm{E}-10$ & NRXN1 \\
\hline Temporal Horn of Lateral Ventricle & 3 & q26.31 & $172,659,246$ & rs10440041 & G & 246.6 & $9.65 \mathrm{E}-09$ & SPATA16 \\
\hline Temporal Horn of Lateral Ventricle & 4 & q32.1 & $156,265,336$ & rs75174989 & G & 258.1 & $3.20 \mathrm{E}-08$ & MAP9 \\
\hline Temporal Horn of Lateral Ventricle & 16 & p12.1 & $27,890,361$ & chr16:27890361:l & TG & 232.2 & 3.63E-08 & GSG1L \\
\hline Precentral & 1 & p36.32 & $5,337,271$ & rs61759358 & $A$ & -718.5 & $2.91 \mathrm{E}-08$ & intergenic \\
\hline Pallidum & 16 & $\mathrm{q} 23.2$ & $80,215,561$ & rs9935652 & G & 109.6 & 4.08E-08 & intergenic \\
\hline Lateral Ventricle & 3 & $\mathrm{p} 12.3$ & $77,856,623$ & rs3852018 & C & 6836 & $4.22 \mathrm{E}-08$ & intergenic \\
\hline Cortex & 3 & $\mathrm{q} 21.3$ & $128,650,296$ & chr3:128650296:l & GTA & 11710 & $4.43 \mathrm{E}-08$ & KIAA1257 \\
\hline Isthmus Cingulate & 22 & $q 13.2$ & $42,398,849$ & rs133310 & C & -179.6 & $5.17 \mathrm{E}-08$ & WBP2NL \\
\hline
\end{tabular}

GWAS results that passed the suggestive significance threshold $(P<5.5 \mathrm{E}-08)$. Bold font: significant after multiple test correction $(P<2.04 \mathrm{E}-09)$.

volume of the temporal (inferior) horns of the lateral ventricles (THLV) and the T allele of $\operatorname{rs} 12467877(P=$ $6.76 \mathrm{E}-10)$, an intronic SNP of the gene Neurexin1 (NRXN1), see Table 2 and Figs. 1 and 2. The association between THLV and the NRXN1 allele was present in both of the two major ethnic groups of this sample $(P=$ $8.195 \mathrm{E}-07$ in Caucasians and $P=9.97 \mathrm{E}-05$ in AfricanAmericans) with no significant allelic frequency differences between them for rs12467877 (MAF 0.13 and 0.09 respectively, ANOVA $P=0.12$, see Suppl. Material), which is consistent with reference data ${ }^{44}$. The $\mathrm{T}$ allele was associated with enlargement of THLV and its effect size on this phenotype was $0.045\left(\mathrm{R}_{\mathrm{G}}^{2}\right)$, that is, $4.5 \%$ of the phenotype variance was attributable to this SNP. However, the identified NRXN1 SNP was not associated with case-control status $(P=0.21$, using PLINK logistic regression model, controlling for sex and ethnicity).

\section{Other suggestive GWAS findings}

Eight more genomic regions had $\mathrm{P}$ values lower than $5.5 \mathrm{E}-08$ with 6 phenotypes; the top 3 were intronic SNPs of genes SPATA16, MAP9 and GSG1L associated with THLV (Table 2).

Precentral, Pallidum and Lateral Ventricle volumes had suggestive associations with intergenic SNPs. A variant in an uncharacterized protein, KIAA1257, was found to be suggestively associated with total cortical volume, and the gene WBP2NL, a sperm domain-binding protein previously associated with intelligence, was associated in our study with the volume of Isthmus Cingulate.

GWAS summary statistics for SNP associations with $P$ $<1 \mathrm{E}-5$ from the 60 brain morphology phenotypes studied are available in Supplementary Material.

\section{Phenotypic associations}

In our sample we observed larger volumes of the temporal (inferior) horns of the lateral ventricles in cases vs. controls (ANOVA $P=0.015$ ). Hippocampal volumes were smaller in cases than in controls (ANOVA $P=$ $4 \mathrm{E}-6)$, and amygdala volumes were also significantly smaller in cases $(P=0.01)$, as reported in previous publications of our group ${ }^{25,45,46}$. Enlargement of THLV was correlated in our sample with volume reduction of the hippocampus and with enlargement of the choroid plexus and caudate (Table 3).

\section{Discussion}

Enlarged ventricular volumes have been among the most consistent anatomical alterations found in SZ and $\mathrm{BD}$, first reported by Johnstone in schizophrenia more than forty years ago ${ }^{47}$. This has been confirmed in SZ and $\mathrm{BD}$ patients compared with healthy controls in large studies by the ENIGMA consortium, and the heritability of the volume of lateral ventricles was calculated to be 0.54 by Kremen et al. ${ }^{48}$. The temporal horn of the lateral ventricle traverses the temporal lobe in a lateral-anterior direction, bordering the caudate, putamen, amygdala, choroid plexus, and hippocampus. Increased ventricular volumes observed in schizophrenia have been attributed to volume reduction of surrounding gray matter structures ${ }^{49,50}$, which is confirmed in this study. Enlargement of choroid plexus in this sample was also associated with cognitive and structural connectivity problems in cases, published by Lizano et al. ${ }^{51}$.

The UK Biobank has published structural MRI GWAS results that contain SNP associations with the temporal horn $^{52}$, which did not replicate our significant finding (UK biobank result for rs12467877 $P=0.095)$. Non-replication of our finding in the UK Biobank sample may be due to a negligible proportion of psychotic patients in their dataset. GWAS analysis of THLV in our controls (only) also gave a non-significant association for rs12467877 $(P=$ 0.07). The Psychiatric Genomic Consortium (PGC) did not find association of case status with rs12467877 


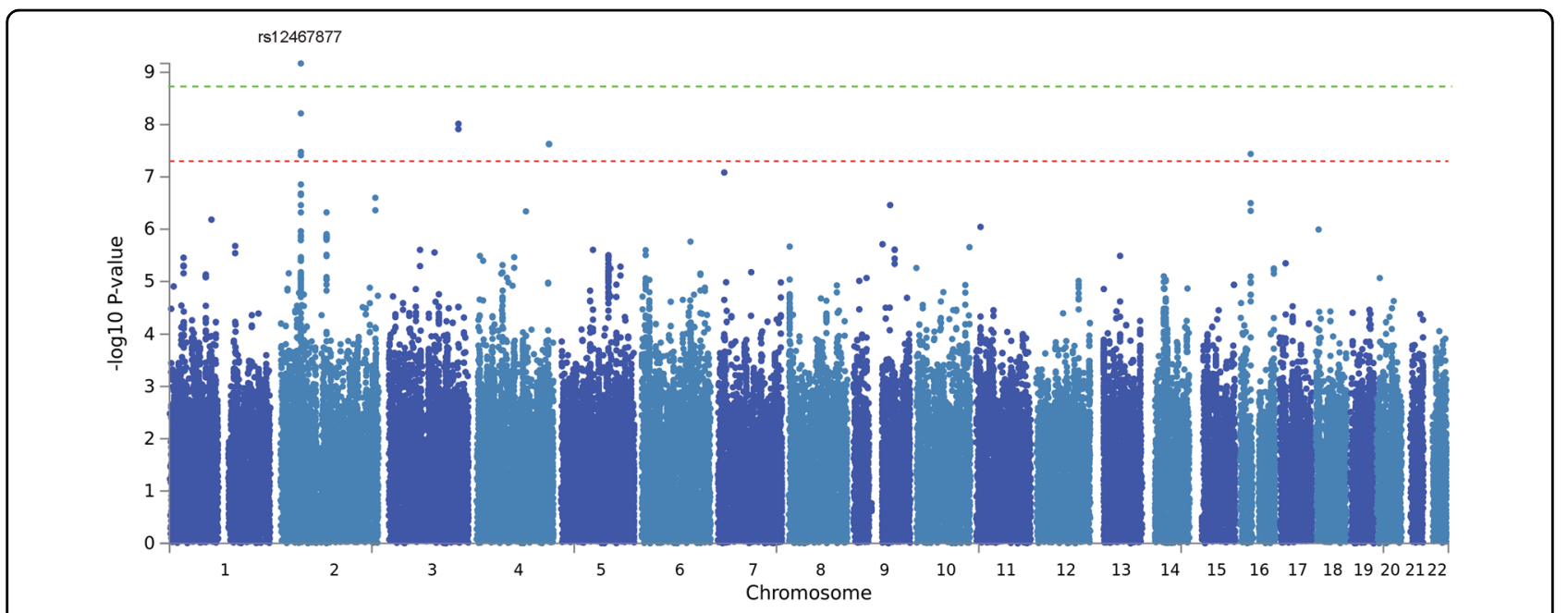

Fig. 1 Genome-wide association of the volume of the temporal horn of the lateral ventricle in the B-SNIP sample. Green dotted line: significance threshold after multiple test correction $(P<2.04 \mathrm{E}-09)$. Red dotted line: suggestive significance threshold $(P<5.56 \mathrm{E}-8)$

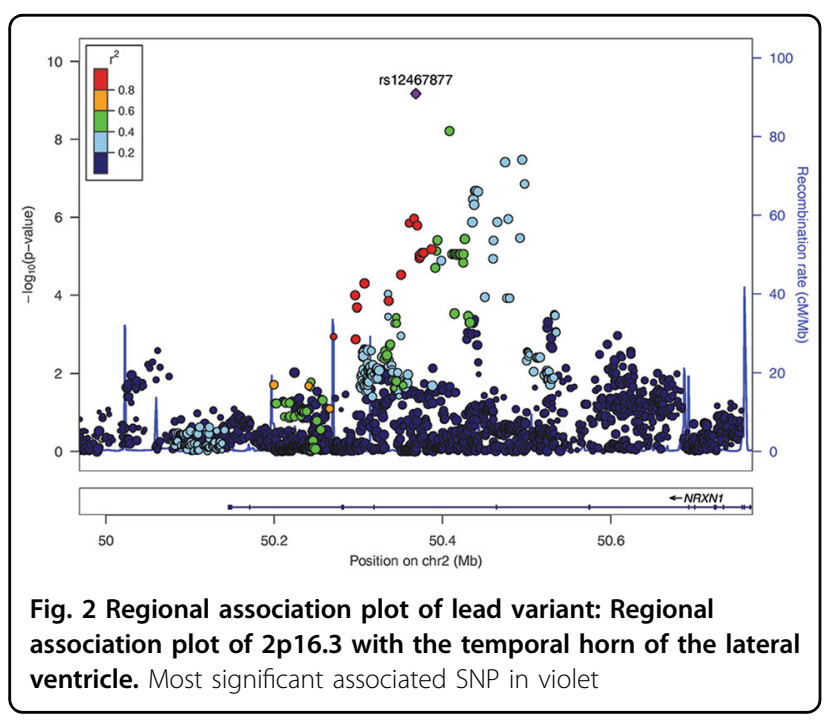

Table 3 Partial correlations of volumes of the combined temporal horns of lateral ventricles and surrounding structures

\begin{tabular}{lcl}
\hline & Correlation & $\mathbf{P}$ \\
\hline Caudate & $\mathbf{0 . 1 0 1}$ & $\mathbf{0 . 0 0 5}$ \\
Putamen & -0.068 & 0.059 \\
Hippocampus & $-\mathbf{0 . 1 5 2}$ & $\mathbf{2 . 0 0 E - 0 5}$ \\
Amygdala & -0.052 & 0.15 \\
Choroid plexus & $\mathbf{0 . 3 5 6}$ & $\mathbf{1 . 6 2 E - 2 4}$ \\
\hline
\end{tabular}

Partial correlations controlling for intracranial volume, age and sex. 2-tailed, 774 df. Bold font: $P<0.05$.
$(P=0.076)$ in their large case-control schizophrenia $\mathrm{GWASs}^{4,53}$, which indicates that the NRXN1 association is pertinent to the intermediate phenotype (THLV volume) and not directly with diagnosis.

NRXN1 encodes a large presynaptic transmembrane protein that binds neuroligins to calcium-dependent synaptic complexes in the central nervous system, and is thus involved in the formation of synaptic contacts ${ }^{54}$. This gene is highly intolerant to loss of function mutations, as described by the Exome Aggregation Consortium (ExAC), with a probability of Loss of function Intolerance, $\mathrm{pLI}=1$ (https://decipher.sanger.ac.uk/genes). Structural copy number variants (CNVs) disrupting NRXN1 are associated with a wide spectrum of brain disorders including schizophrenia, autism, and developmental disorders ${ }^{55-57}$. The UK Brain Expression Consortium (http://www. braineac.org $/)^{41}$ has reported expression data for NRXN1 related to rs12467877, with no significant evidence for affecting expression (see Suppl. Material, page 10). The Genotype-Tissue Expression (GTEx) project (http://gtexportal.org) also does not report functional associations for rs12467877. Functional analyses using FUMA identified this and other suggestively associated SNPs to be related to the expression of other genes, such as PPP1R21 and GSG1L (Suppl. Material), which are highly expressed in brain during development and adulthood.

Three other genes suggestively associated with THLV are SPATA16, MAP9 and GSG1L. SPATA16 is involved in spermatogenesis and structural variants of this gene have been associated with globozoospermia ${ }^{58}$. MAP9 (also known as ASAP) is a gene encoding a microtubule associated protein highly expressed in brain and thyroid 
(https://gtexportal.org) ${ }^{59}$. The germ cell-specific gene 1like protein (GSG1L) is a component of the AMPA receptor complex, a glutamate transmembrane receptor in the CNS, highly expressed in nucleus accumbens and basal ganglia, that has been associated with synaptic plasticity, mathematical ability, and creative activities in music $^{60-62}$. Functional analysis of the GSG1L suggestively associated SNPs identified additional eQTLs (See Supplementary Material).

This is the first report of a common SNP variant of NRXN1 associated with enlargement of THLV volume in psychosis, and this volume is correlated with reduction of the hippocampus and enlargement of the caudate and choroid plexus. The association $p$-value $6.76 \mathrm{E}-10$ is significant after multiple test correction, but further research with a larger sample of cases and controls will be needed to confirm these results. Although the role of this common NRXN1 SNP in psychosis is indirect, this study adds evidence to the role of NRXN1 in psychosis, which was originally implied by rare CNVs of this gene.

\section{Acknowledgements}

Thanks to the probands, their families and volunteers who joined this study and contributed their time and individual data. Thanks also to Prof. Carlos Morcillo, who kindly customized his program Manhattan Plotter for this study. This work made use of the Bionimbus Protected Data Cloud (PDC), which is a collaboration between the University of Chicago Center for Data Intensive Science (CDIS) and the Open Commons Consortium (OCC). The Bionimbus PDC allows users authorized by $\mathrm{NIH}$ to compute over human genomic data in a secure compliant fashion. The Bionimbus PDC is part of the OSDC ecosystem.

\section{Author details}

${ }^{1}$ University of Chicago, Department of Psychiatry and Behavioral Neurosciences, Chicago, USA. ${ }^{2}$ Massachusetts Institute of Technology, Cambridge, USA. ${ }^{3}$ Harvard Medical School, Department of Genetics, Boston, USA. ${ }^{4}$ Stanley Center, Broad Institute of MIT and Harvard, Cambridge, USA. ${ }^{5}$ Harvard Medical School, Department of Psychiatry, Boston, USA. ${ }^{6}$ University of Chicago Laboratory for Advanced Computing, Chicago, USA. ${ }^{7}$ Harvard University, Cambridge, USA. ${ }^{8}$ Boston University, Boston, USA. ${ }^{9}$ Child Psychiatry Branch, National Institutes of Mental Health, National Institutes of Health, Bethesda, MD, USA. ${ }^{10}$ Broad Institute of MIT and Harvard, Cambridge, USA. ${ }^{11}$ University of Minnesota, Department of Experimental and Clinical Pharmacology and Department of Psychiatry, Minneapolis, USA. ${ }^{12}$ Rosalind Franklin University, North Chicago, USA. ${ }^{13}$ Northwestern University, Evanston, USA. ${ }^{14}$ University of Muenster, Munster, Germany. ${ }^{15}$ Department of Psychology, University of Georgia, Athens, Georgia. ${ }^{6}$ Virginia Commonwealth University, Richmond, USA. ${ }^{17}$ Yale University Departments of Psychiatry \& Neuroscience, New Haven, USA. ${ }^{18}$ University of Texas Southwestern Medical Center, Department of Psychiatry, Dallas, USA. ${ }^{19}$ University College London and Centre for Psychiatry, Barts and the London School of Medicine and Dentistry, London, UK. ${ }^{20}$ Rush University Medical Center, Chicago, USA. ${ }^{21}$ SUNY Upstate Medical University, Binghamton, USA. ${ }^{22}$ University of Chicago, Department of Human Genetics, Chicago, USA

\section{Data availability}

Manhattan plots and GWAS summary statistics for SNP associations with $P<$ $1 \mathrm{E}-5$ from the 60 brain morphology phenotypes studied here are available in Supplementary Material. Complete GWAS summary statistics can be requested from the authors.

\section{Conflict of interest}

Dr. Keshavan has received a grant from Sunovion and is a consultant to Forum Pharmaceuticals. Dr. Tamminga is a consultant to IntracellularTherapies, an ad hoc consultant to Takeda and Astellas and received a grant from Sunovion. The rest of the authors declare that they have no conflict of interest.

\section{Publisher's note}

Springer Nature remains neutral with regard to jurisdictional claims in published maps and institutional affiliations.

Supplementary Information accompanies this paper at (https://doi.org/ 10.1038/s41398-019-0564-9).

Received: 21 March 2019 Revised: 11 July 2019 Accepted: 30 July 2019 Published online: 17 September 2019

\section{References}

1. Padmanabhan, J. L. et al. Correlations between brain structure and symptom dimensions of psychosis in schizophrenia, schizoaffective, and psychotic bipolar I disorders. Schizophr. Bull. 41, 154-162 (2015).

2. Skudlarski, P. et al. Diffusion tensor imaging white matter endophenotypes in patients with schizophrenia or psychotic bipolar disorder and their relatives. Am. J. Psychiatry 170, 886-898 (2013).

3. Meda, S. A. et al. Multivariate analysis reveals genetic associations of the resting default mode network in psychotic bipolar disorder and schizophrenia. Proc. Natl Acad. Sci. USA 111, E2066-E2075 (2014).

4. Schizophrenia Working Group of the Psychiatric Genomics Consortium. Biological insights from 108 schizophrenia-associated genetic loci. Nature 511, 421-427 (2014).

5. Consortium., InternationalSchizophrenia et al. Common polygenic variation contributes to risk of schizophrenia and bipolar disorder. Nature 460, 748-752 (2009).

6. Cross-Disorder Group of the Psychiatric Genomics Consortium. Identification of risk loci with shared effects on five major psychiatric disorders: a genomewide analysis. Lancet 381, 1371-1379 (2013).

7. Swerdlow, N. R., Gur, R. E. \& Braff, D. L. Consortium on the Genetics of Schizophrenia (COGS) assessment of endophenotypes for schizophrenia: an introduction to this Special Issue of Schizophrenia Research. Schizophr. Res. 163, 9-16 (2015)

8. Hager, B. M. \& Keshavan, M. S. Neuroimaging biomarkers for psychosis. Curr. Behav. Neurosci. Rep. 2015, 1-10 (2015).

9. Gray, B. E. et al. Detecting reliable cognitive change in individual patients with the MATRICS Consensus Cognitive Battery. Schizophr. Res. 159, 182-187 (2014)

10. McDonald, C. The Maudsley Family Study of Psychosis: A Quest for Intermediate Phenotypes. (pp. 218. Psychology Press, Hove England; New York, 2008).

11. Gottesman, I. I. \& Gould, T. D. The endophenotype concept in psychiatry: etymology and strategic intentions. Am. J. Psychiatry 160, 636-645 (2003).

12. Gottesman, I. I. \& Shields, J. Genetic theorizing and schizophrenia. Br. J. Psychiatry 122, 15-30 (1973).

13. Gershon, E. S., Dunner, D. L. \& Goodwin, F. K. Toward a biology of affective disorders. Genetic contributions. Arch. Gen. Psychiatry 25, 1-15 (1971).

14. Rieder, R. O. \& Gershon, E. S. Genetic strategies in biological psychiatry. Arch. Gen. Psychiatry 35, 866-873 (1978).

15. Franke, B. et al. Genetic influences on schizophrenia and subcortical brain volumes: large-scale proof of concept. Nat. Neurosci. 19, 420-431 (2016).

16. Strasser, H. C. et al. Hippocampal and ventricular volumes in psychotic and nonpsychotic bipolar patients compared with schizophrenia patients and community control subjects: a pilot study. Biol. Psychiatry 57, 633-639 (2005).

17. Hibar, D. P. et al. Subcortical volumetric abnormalities in bipolar disorder. Mol. Psychiatry 21, 1710-1716 (2016).

18. Roy, P. D. et al. Temporal horn enlargement is present in schizophrenia and bipolar disorder. Biol. Psychiatry 44, 418-422 (1998).

19. Bush, W. S. \& Moore, J. H. Chapter 11: Genome-wide association studies. PLoS Comput Biol. 8, e1002822 (2012).

20. Dahl, A. et al. A multiple-phenotype imputation method for genetic studies Nat. Genet 48, 466-472 (2016).

21. Tamminga, C. A. et al. Bipolar and schizophrenia network for intermediate phenotypes: outcomes across the psychosis continuum. Schizophr. Bull. $\mathbf{4 0}$ (Suppl 2), S131-S137 (2014). 
22. Tamminga, C. A. et al. Clinical phenotypes of psychosis in the BipolarSchizophrenia Network on Intermediate Phenotypes (B-SNIP). Am. J. Psychiatry 170, 1263-1274 (2013).

23. Fischl, B. FreeSurfer. Neuroimage 62, 774-781 (2012).

24. Desikan, R. S. et al. An automated labeling system for subdividing the human cerebral cortex on MRI scans into gyral based regions of interest. Neuroimage 31, 968-980 (2006).

25. Ivleva, E. I. et al. Brain structure biomarkers in the psychosis biotypes: findings from the bipolar-schizophrenia network for intermediate phenotypes. Biol. Psychiatry 82, 26-39 (2017).

26. Sun, L. \& Dimitromanolakis, A. PREST-plus identifies pedigree errors and cryptic relatedness in the GAW18 sample using genome-wide SNP data. BMC Proc. 8 , S23 (2014). Suppl 1 Genetic Analysis Workshop 18Vanessa Olmo.

27. Manichaikul, A. et al. Robust relationship inference in genome-wide association studies. Bioinformatics 26, 2867-2873 (2010)

28. Genomes Project, C. et al. An integrated map of genetic variation from 1,092 human genomes. Nature 491, 56-65 (2012).

29. Williams, A. L., Patterson, N., Glessner, J., Hakonarson, H. \& Reich, D. Phasing of many thousands of genotyped samples. Am. J. Hum. Genet. 91, 238-251 (2012).

30. Howie, B., Fuchsberger, C., Stephens, M., Marchini, J. \& Abecasis, G. R. Fast and accurate genotype imputation in genome-wide association studies through pre-phasing. Nat. Genet. 44, 955-959 (2012).

31. Howie, B. N., Donnelly, P. \& Marchini, J. A flexible and accurate genotype imputation method for the next generation of genome-wide association studies. PLoS Genet. 5, e1000529 (2009).

32. Chang, C. C. et al. Second-generation PLINK: rising to the challenge of larger and richer datasets. Gigascience 4, 7 (2015).

33. Purcell, S. et al. PLINK: a tool set for whole-genome association and population-based linkage analyses. Am. J. Hum. Genet. 81, 559-575 (2007).

34. Heath, A. P. et al. Bionimbus: a cloud for managing, analyzing and sharing large genomics datasets. J. Am. Med Inf. Assoc. 21, 969-975 (2014).

35. Kent, W. J. et al. The human genome browser at UCSC. Genome Res. 12 996-1006 (2002).

36. Watanabe, K., Taskesen, E., van Bochoven, A. \& Posthuma, D. Functional mapping and annotation of genetic associations with FUMA. Nat. Commun. 8 , 1826 (2017)

37. Pruim, R. J. et al. LocusZoom: regional visualization of genome-wide association scan results. Bioinformatics 26, 2336-2337 (2010).

38. Price, A. L. et al. Principal components analysis corrects for stratification in genome-wide association studies. Nat. Genet 38, 904-909 (2006).

39. Carithers, L. J. et al. A Novel Approach to High-Quality Postmortem Tissue Procurement: The GTEx Project. Biopresen. Biobank 13, 311-319 (2015).

40. Miller, J. A. et al. Transcriptional landscape of the prenatal human brain. Nature 508, 199-206 (2014).

41. Ramasamy, A. et al. Genetic variability in the regulation of gene expression in ten regions of the human brain. Nat. Neurosci. 17, 1418-1428 (2014).

42. Li, M. X., Yeung, J. M., Cherny, S. S. \& Sham, P. C. Evaluating the effective numbers of independent tests and significant p-value thresholds in commercial genotyping arrays and public imputation reference datasets. Hum. Genet. 131, 747-756 (2012).

43. Li, J. \& Ji, L. Adjusting multiple testing in multilocus analyses using the eigenvalues of a correlation matrix. Heredity 95, 221-227 (2005).
44. Marcus, J. H. \& Novembre, J. Visualizing the geography of genetic variants. Bioinformatics 33, 594-595 (2017).

45. Mathew, l. et al. Medial temporal lobe structures and hippocampal subfields in psychotic disorders: findings from the Bipolar-Schizophrenia Network on Intermediate Phenotypes (B-SNIP) study. JAMA Psychiatry 71, 769-777 (2014)

46. Arnold, S. J. et al. Hippocampal volume is reduced in schizophrenia and schizoaffective disorder but not in psychotic bipolar I disorder demonstrated by both manual tracing and automated parcellation (FreeSurfer). Schizophr. Bull. 41, 233-249 (2015).

47. Johnstone, E. C., Crow, T. J., Frith, C. D., Husband, J. \& Kreel, L. Cerebral ventricular size and cognitive impairment in chronic schizophrenia. Lancet $\mathbf{2}$ 924-926 (1976).

48. Kremen, W. S. et al. Heritability of brain ventricle volume: converging evidence from inconsistent results. Neurobiol. Aging 33, 1-8 (2012).

49. Gaser, C., Nenadic, I., Buchsbaum, B. R., Hazlett, E. A. \& Buchsbaum, M. S. Ventricular enlargement in schizophrenia related to volume reduction of the thalamus, striatum, and superior temporal cortex. Am. J. Psychiatry 161, 154-156 (2004).

50. Degreef, G. et al. Volumes of ventricular system subdivisions measured from magnetic resonance images in first-episode schizophrenic patients. Arch. Gen. Psychiatry 49, 531-537 (1992).

51. Lizano P. L., et al. Choroid plexus enlargement is associated with cognitive inflammatory and structural phenotypes across the psychosis spectrum. Am. J. Psychiatry. 176, 564-572 (2019).

52. Elliott, L. T. et al. Genome-wide association studies of brain imaging phenotypes in UK Biobank. Nature 562, 210-216 (2018).

53. Bipolar Disorder and Schizophrenia Working Group of the Psychiatric Genomics Consortium. Genomic Dissection of Bipolar Disorder and Schizophrenia, Including 28 Subphenotypes. Cell 173, 1705-1715 e1716 (2018).

54. NCBI Resource Coordinators. Database Resources of the National Center for Biotechnology Information. Nucleic Acids Res. 45, D12-D17 (2017).

55. Schaaf, C. P. et al. Phenotypic spectrum and genotype-phenotype correlations of NRXN1 exon deletions. Eur. J. Hum. Genet. 20, 1240-1247 (2012).

56. Duong, L. et al. Mutations in NRXN1 in a family multiply affected with brain disorders: NRXN1 mutations and brain disorders. Am. J. Med Genet B Neuropsychiatr. Genet. 159B, 354-358 (2012).

57. Rujescu, D. et al. Disruption of the neurexin 1 gene is associated with schizophrenia. Hum. Mol. Genet. 18, 988-996 (2009).

58. Dam, A. H. et al. Homozygous mutation in SPATA16 is associated with male infertility in human globozoospermia. Am. J. Hum. Genet. 81, 813-820 (2007).

59. GTEx Consortium et al. Genetic effects on gene expression across human tissues. Nature 550, 204-213 (2017).

60. Lee, J. J. et al. Gene discovery and polygenic prediction from a genome-wide association study of educational attainment in 1.1 million individuals. Nat. Genet. 50, 1112-1121 (2018).

61. Gu, X. et al. GSG1L suppresses AMPA receptor-mediated synaptic transmission and uniquely modulates AMPA receptor kinetics in hippocampal neurons. Nat. Commun. 7, 10873 (2016).

62. Oikkonen, J. et al. Creative activities in music-a genome-wide linkage analysis. PLOS ONE 11, e0148679 (2016). 